\title{
POWER INPUT AND DISSIPATION IN THE POSITIVE COLUMN OF A CESIUM DISCHARGE
}

\author{
By F. L. Mohler
}

ABSTRACT

The power input per centimeter is equal to the voltage gradient times the current. Two recognized sources of power dissipation are the recombination of ions on the tube walls and the atomic radiation.

The voltage gradient is measured between two probes at the axis of the discharge tube. The flow of ions to the walls is measured by a disk probe at the wall. The recombination energy is the kinetic energies of the ions and electrons, which can be evaluated from the probe current-voltage curves, plus the ionization energy.

The total radiation was measured by a thermopile while transmission corrections were determined by a photo-electric cell. The first doublet of the principal series at 8,521 and 8,944 A contributes most of the radiation.

Results show that with low currents and pressures the radiation and recombination losses account for nearly the entire power input. With increasing currents and pressures there is an increasing balance of power which is unaccounted for. All the power terms change very slowly with pressure. The recombination loss is negligible at low currents and the most important factor at high currents.

\section{CONTENTS}

II. Electrical measurements _.....

III. Radiation measurements 30

IV. Results _... 31

V. Conclusion

\section{INTRODUCTION}

Conditions in the positive column are determined by the nature of the gas in the tube, the density of the gas, the size of the tube and the current. This applies to all types of direct current discharge; cold cathode glow discharges, thermionic discharges and arcs when they operate under current and pressure conditions in which a true column exists. In the uniform or unstriated column the voltage gradient, electron and ion concentrations, electron speed, and, indeed, all the dependent variables remain nearly constant along the length of the column. For this reason it is the simplest part of the discharge to study, and it should be the simplest to understand though there is no general theory relating all dependent and independent variables. $^{1}$

The power input per centimeter of a uniform column is equal to the voltage gradient times the current, and it follows from the nature of the column that the power dissipated in each element of length is equal to the input. The dissipation of power appears as heating of the tube walls, either directly or by conduction and radiation from 
the gas, and as selective radiation of the atoms which is transmitted by the tube walls. The general statement is made in the Handbuch der Physik that the total radiation is a small fraction of the power input, but the experiments cited deal with gases which have their strongest radiation in the far ultra-violet and this is completely absorbed by the tube walls. Thus Crew and Hulburt ${ }^{2}$ find that the total radiation emitted by a hydrogen discharge is about 1 per cent of the input, but they point out that about nine times the observed radiation is in the first Lyman line alone.

The alkali vapor spectra are the only spectra in which all the strong lines are transmitted by glass. The possibility of obtaining a high radiant efficiency is utilized in the Osram sodium lamp. ${ }^{3}$ Under normal operating conditions (current density 1 amp. per $\mathrm{cm}^{2}$ ) the lamp radiates about 9 per cent of the power input. This radiation is predominantly $D$ lines and the luminous efficiency is probably higher than any other light source, though the figure for radiation efficiency is certainly not impressive. The fact is that the lamp has to be overloaded to a point of low efficiency to maintain a high tube temperature and vaporize the sodium. With one-tenth of the normal current and external heating a radiation efficiency of 70 per cent is observed.

There is no published work in which radiation measurements have been combined with detailed electrical measurements in the study of a discharge. Other phases of power dissipation have been treated by Langmuir and his associates in their comprehensive theoretical ${ }^{4}$ and experimental ${ }^{5}$ studies of the mercury positive column. An important factor is the flow of ions to the walls and their recombination on the walls. The fraction of the power dissipated in this way depends on the current and is small for low currents. Elastic collisions between electrons and atoms will give some energy to the atoms, but Killian has evaluated this loss and finds it negligibly small for the mercury arc. He concludes that power dissipated in inelastic collisions between electrons and atoms and radiated by the atoms must account for the balance of the power loss not accounted for by recombination on the walls. The conclusion seems entirely reasonable, though there is a possibility that the excited atoms dissipate some energy by collisions with normal atoms. The probability of dissipation of energy by collision (quenching) during the life of an excited state is undoubtedly small under most discharge conditions; but since the resonance radiation is very strongly absorbed, the radiation diffuses slowly through the gas by repeated absorption and emission. For this reason the small probability of quenching during the radiation life must be multiplied by a large and unknown factor to give the probability of quenching during the diffusion time.

This paper reports measurements of power input, radiation loss, and wall recombination loss in the positive column of a cæsium discharge. Tubes of one diameter $1.8 \mathrm{~cm}$ have been used, and the effect of the two remaining independent variables, vapor density, and current, have been studied. This work has been done in conjunction with a study of collision processes in the positive column which will be reported in another paper.

\footnotetext{
${ }^{2}$ Crew and Hulburt, Phys. Rev., vol. 29, p. 843, 1927.

3 Kreft, Pirani, and Rompe, Tech. Wissenschaft Abhandl aus dem Osram Konzern, vol 2, p. 24, 1931.

4 Tonks and Langmuir, General Theory of the Plasma of an Arc, Phys. Rev., vol. 34, p. 876, 1929.

- Killian, Uniform Positive Column of an Electric Discharge in Mercury Vapor, Phys. Rev., vol. 35, p. 1238,1930 .
} 


\section{ELECTRICAL MEASUREMENTS}

Figure 1 illustrates the type of discharge tube used. A thermionic discharge from an oxide-coated platinum strip cathode was employed. Vapor pressure was controlled by a separate heater around the side tube containing the cæsium. Tubes were outgassed by baking and by running a discharge for four or five hours before sealing off from the pumps.

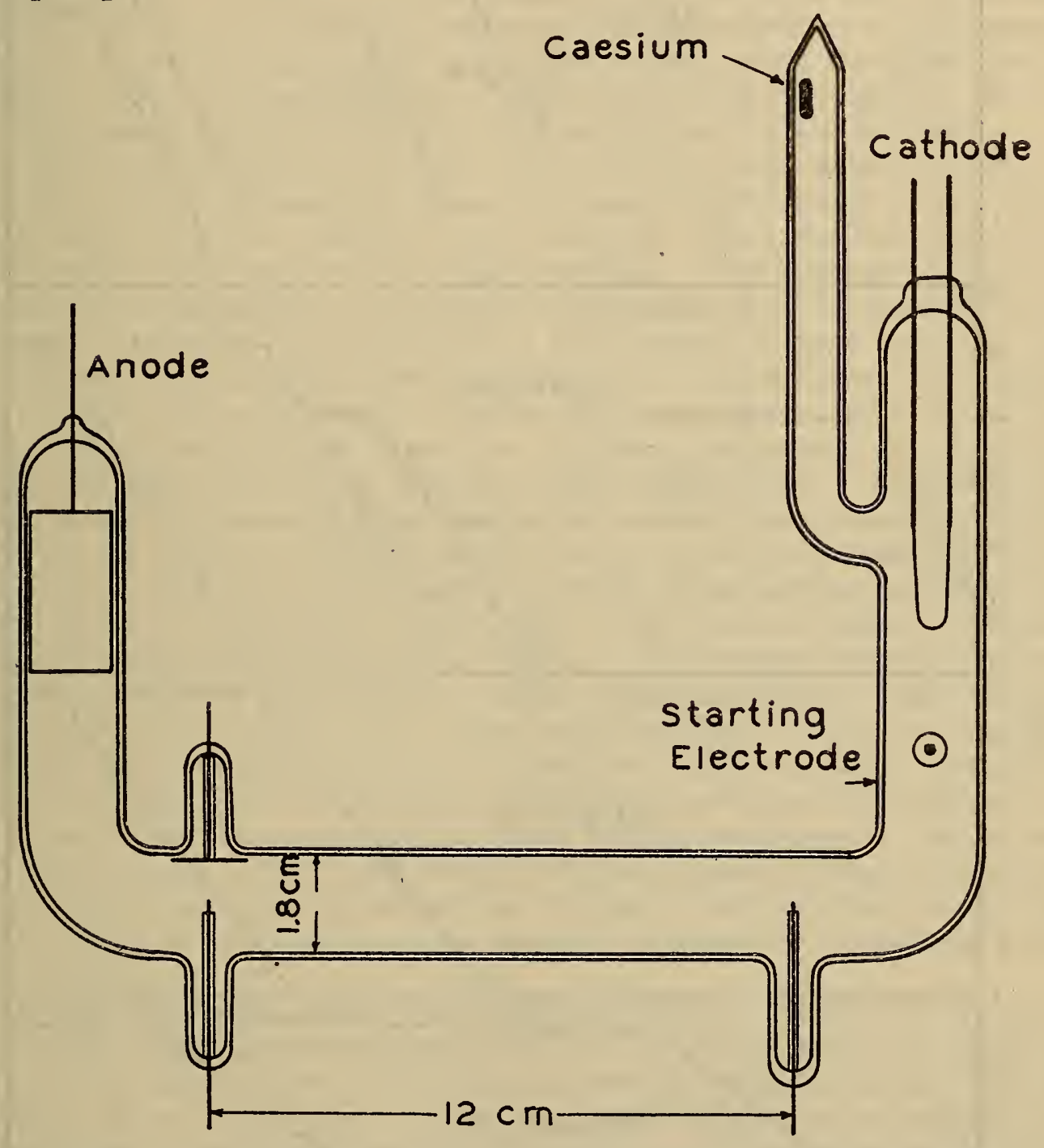

FigURe 1.-Discharge tube used in making power input and dissipation measurements

The voltage gradient was measured between two small probes near the axis of the tube and $12 \mathrm{~cm}$ apart. For the lower pressure and current conditions the probes were platinum wires $0.4 \mathrm{~mm}$ in diameter and $2 \mathrm{~mm}$ long. For the higher currents the platinum wires were cut off flush with the insulating tube. In cæsium vapor difficulty is encountered from leakage over the insulating surfaces, and this was greatly reduced by avoiding a close fit between the insulating tube and the wire. Melting off of the small probes by the striking of an arc was a source of trouble. If a probe is too large the current may rise to a destructive value even at potentials negative to the space potential. The random space current becomes so large that 
an electrode less than $0.01 \mathrm{~cm}^{2}$ in area may rob the discharge and suddenly become the anode. The ion current to the walls was measured by a disk of platinum $1 \mathrm{~cm}$ in diameter which was bent to fit against the wall.

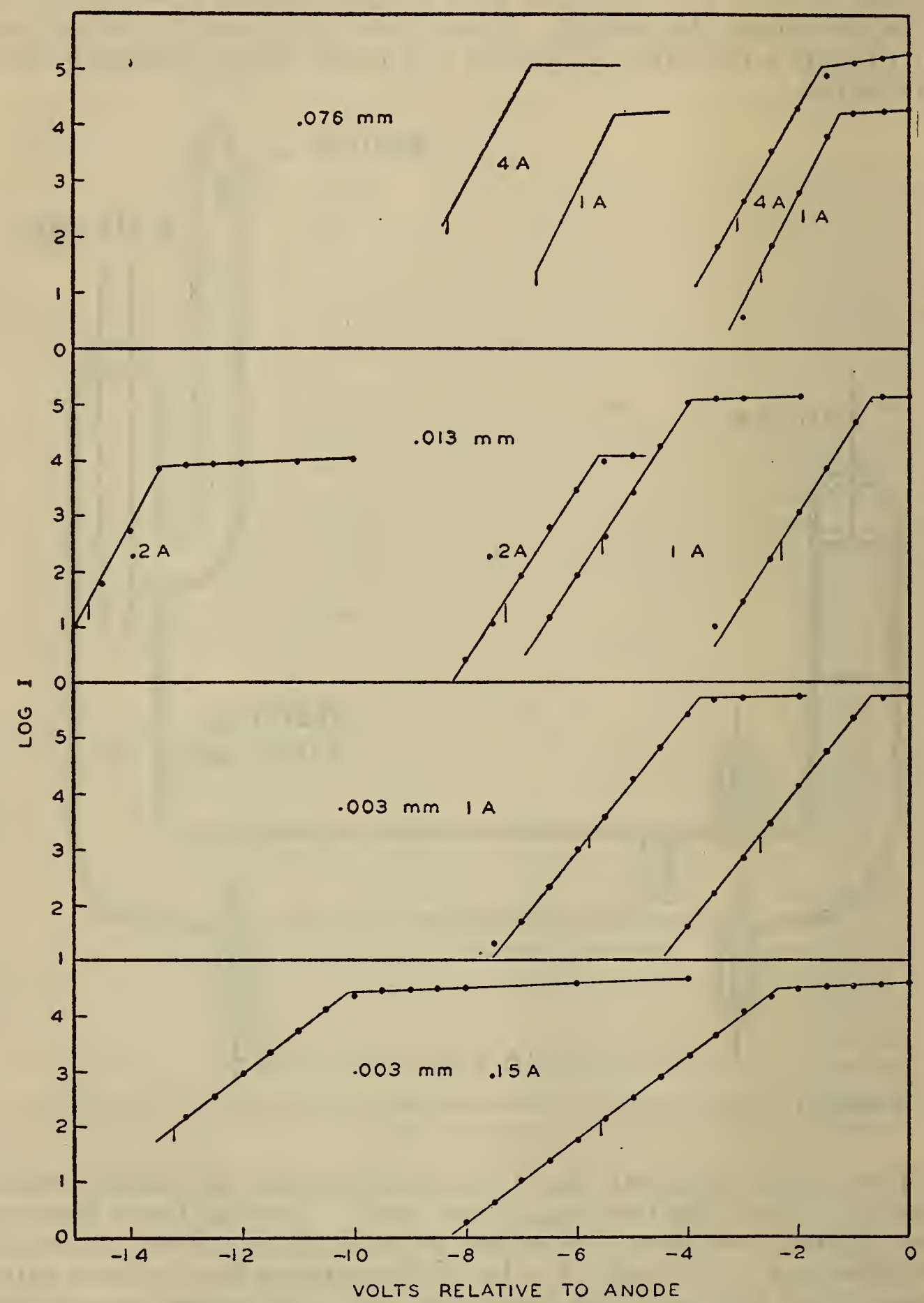

FIGURE 2.-Log current voltage curves for the two small probes at the axis of the tube and $12 \mathrm{~cm}$ apart

Vertical dashes mark the potentials of zero current.

Figure 2 gives plots of the log of the electron current to the small probes versus potential relative to the anode for several typical conditions. The plots are accurately linear for all conditions. The slope 
of the left branch measures the electron energy; the intersection of the two branches measures the space potential. The slopes as measured by the two probes are equal within experimental error. The electron current to the disk also rises at the same rate, showing that the electron energy is the same at the center and at the wall. This has been shown before by Killian. ${ }^{6}$

The voltage difference between the probes can be obtained from the difference between the space potentials, or since the curves are parallel, it can be measured between the potentials of zero current. The two intervals agree within about 0.1 volt, while the difference is several volts. This difference, divided by 12 and multiplied by the current, gives the input in watts per centimeter of the column.

The power dissipated by flow of ions to the tube walls is equal to the ion current in amperes times the sum of the ionization potential (3.9 volts) plus the potential drop through which the ions fall, plus the energy in electron volts of the electrons. The leakage to the disk at negative potentials was always measurable, but in general small enough so that an extrapolation of the current voltage curve to the wall potential should involve no serious error. This current multiplied by the geometrical factor, 7.2 , gives the ion current to $1 \mathrm{~cm}$ of the tube. The assumption that the current, corrected for leakage, measures pure ion current requires some justification. In many discharges the electron emission from negative electrodes is an important part of the current. This emission comes from photo-electric effect and from collision of excited atoms with the metal surface and in either case depends on whether the energy of the excited states is greater than the work function of the metal. The energy of the cæsium resonance state is 1.45 electron volts. Ives and Olpin ${ }^{7}$ find that the minimum work function of a pure metal surface in cæsium is about equal to this, while the results of Boeckner and the author ${ }^{8}$ on probe radiation indicate that platinum probes in a cæsium discharge have a high work function compared to other metals (well above 2 volts, but not measured). The consistency of the present measurements of power input and loss gives further evidence that no serious error has been made in interpreting the current at negative potentials as an ion current.

In the discharge space the random electron current is about a thousand times the ion current, and the insulating wall must take up a potential which draws the full ion current to it and limits the electron current to an equal value. The potential difference is limited to a thin sheath a fraction of a millimeter thick over the surface of the tube. There is also a small potential difference in the same direction between the space potential near the wall and at the center of the tube. Since the electrons have a Maxwell distribution of velocity, the small fraction of fast electrons which pass through the retarding field will reach the wall with the same distribution of velocity as the electrons in the field free space. The contribution of the electorn kinetic energy in electron volts ( 0.2 to 0.3 volt) is obtained from the semilog. plot.

$$
V_{0}=\left(V_{2}-V_{1}\right) /\left(\ln I_{1}-\ln I_{2}\right)
$$

A rigorous measurement of the potential through which the ions fall

6 Killian, Phys. Rev., vol. 35, p. 1238, 1930.

7 Ires and Olpin, Phys. Rev., vol. 34, p. 117, 1929.

${ }^{8}$ Mohler and Boeckner, B. S. Jour. Research, vol. 7, p. 751, 1931. 
would require a small probe at the wall which could be brought to the space potential. (The disk is much too big for this.) Then the difference between the potential of zero current and the space potential would give the potential drop in the sheath (about 2 volts). The average ion also falls through a potential drop somewhat less than the difference in space potentials near the walls and at the center of the tube, a matter of a few tenths of a volt. From a consideration of Killian's measurements it was evident that the difference between the space potential and potential of zero current at the center of the the tube was slightly larger than the potential drop across the wall sheath and less than the drop between the center of the tube and the wall. The error in using this potential drop instead of the true potential difference can only be 0.1 or 0.2 volt; and as the recombination energy is 6 or 7 electron volts, the use of another probe at the wall seemed unnecessary.

\section{RADIATION MEASUREMENTS}

The predominant lines in an alkali metal spectrum are the first doublet in the principal series, and in cæsium these lines fall in the near infra-red at 8,521 and 8,944 A. Nearly all other lines fall in a range transmitted by glass and water except the first doublet of the diffuse series near 36,000 and 35,000 A. As the tube is operated in a furnace at $250^{\circ}$ to $300^{\circ} \mathrm{C}$., the use of a water cell in making thermopile measurements was unavoidable and this far infra-red doublet was completely filtered out. The G. E. cæsium-oxygen-silver photoelectric cell is very sensitive to the resonance lines, and it was useful in evaluating the transmission of the water cell, tube walls, etc. A disk thermopile $0.5 \mathrm{~cm}$ in diameter was used.

The procedure was as follows: The thermopile was calibrated without the water cell or any window against a radiation standard. It was then exposed through a water cell and furnace window to the cæsium radiation from the full width and a $10 \mathrm{~cm}$ length of the positive column. The galvanometer deflection of 3 or $4 \mathrm{~cm}$ could be measured with sufficient precision by averaging many observations. It was inconveniently small for routine measurements, so the deflection of the direct radiation was compared with the deflection produced when the radiation from a $1 \mathrm{~cm}$ length of the tube was focused by a short focus lens so that the entire image fell on the thermopile. The transmission of the tube wall, furnace window, and water cell for the cæsium radiation were measured by the photo-electric cell. It was assumed that the radiation from the discharge was equal in all directions. Two independent calibrations before and after the series of experiments gave values of $2.13 \times 10^{-2}$ and $2.18 \times 10-^{2}$ watts per centimeter galvanometer deflection per centimeter of the column for the total radiation.

An experiment was made on the absorption of the cæsium radiation by a $4 \mathrm{~cm}$ cell of cæsium vapor. With the discharge vapor pressure $0.004 \mathrm{~mm}$, the cell containing vapor at about ten times that pressure reduced the photo-electric effect to less than one-tenth. As the vapor is perfectly transparent to all lines except the principal series lines, this shows that most of the energy is in the resonance lines. Photographs of the spectrum indicate that these lines remain predominant at all pressures. Radiation measurements were made before and after 
a series of electrical measurements with no evidence of any error appreciable in comparison with the electrical measurements or with the error in optical adjustments when tubes were changed.

\section{RESULTS}

Discharges operated with a voltage drop ranging from 5 to 15 volts. It was found that a large change in the battery voltage with a compensating change in the series resistance to give equal current did not change the radiation by 0.1 per cent. Also a change in the voltage drop in the tube produced by changing the cathode temperature had no effect. Currents ranged from 0.15 to 4 amperes; pressures from 0.001 to $0.3 \mathrm{~mm}$. The low pressure limit was set by the dark space approaching the probe nearest the cathode.

Figures 3 and 4 show the power input; $P$, the power dissipated by recombination; $W$, the radiation; $R$, and $P-W$ as functions of the pressure and the current. With currents of $0.2 \mathrm{amp}$. practically all the power input is radiated. With currents of 1 amp. or less and pressures less than $0.03 \mathrm{~mm}, P-W$ is nearly equal to $R$; that is, the power input is nearly all accounted for. The mean value for all measurements in this range gives

$$
R /(P-W)=0.95 \pm 0.04
$$

where 0.04 is the mean error and does not include the calibration error. With increasing current and pressure there is a rapidly increasing difference between $P-W$ and $R$. A plot of this unexplained residual is given as a function of the current for various pressures in Figure

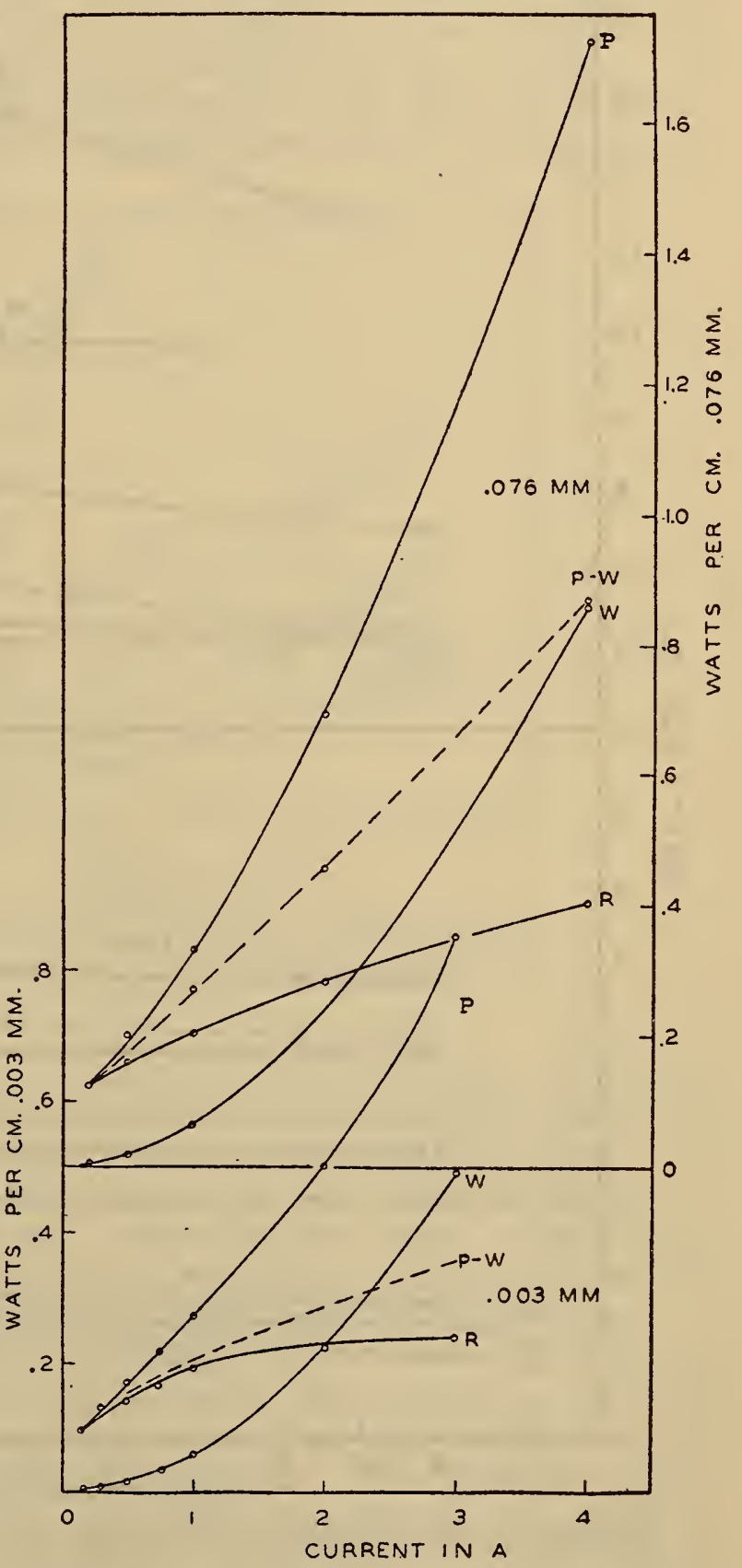

FIGURE 3.-Power terms as a function of current ( $P$, input; $W$, recombination; $R$, radiation.) 5. Up to $0.013 \mathrm{~mm}$ the residual is independent of the pressure, but above this point increases roughly in proportion to the pressure. The experimental uncertainty is relatively large for this residual. 


\section{CONCLUSIONS}

Little can be said as yet about the factors determining the form of the curves of Figures 3, 4, and 5. With increasing current the gradient at first decreases and then increases and correspondingly the input curve of Figure 3 changes. The rapid increase at higher currents

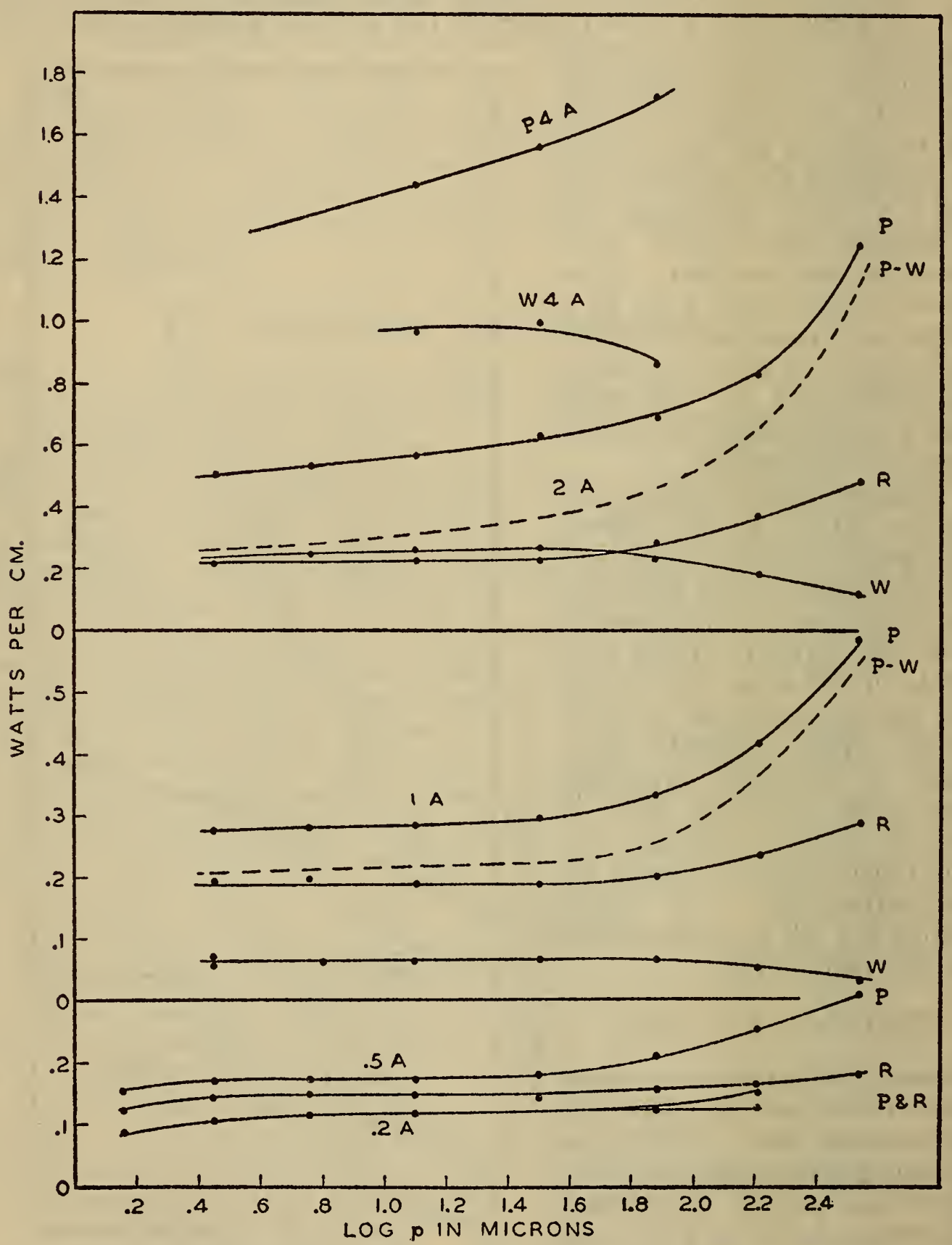

Figure 4.-Power terms as a function of the log of the pressure in microns

comes in part from the recombination loss and in part from the unknown residual. The variation in recombination loss, $W$, depends largely on the ion current, for the energy per ion changes very slowly. The ion current is roughly proportional to the electron concentration. The striking feature of Figure 4 is the very slow variation of all power terms with pressure. The measurements cover a 300 -fold range of 
pressure, and over half of this range the variation is scarcely more than the experimental error. An appreciable variation is evident with high pressures and high currents, and the unknown residual effect is an important factor in this. The fact that there is an unexplained balance in the power equation is not surprising. We have mentioned in the introduction several other sources of power loss; the unmeasured far infra-red radiation, the dissipation of energy by elastic collisions, and the quenching of resonance radiation by interatomic collisions, and there are undoubtedly other possibilities. The evidence is as yet insufficient to choose between them. It is reassuring to find that over a considerable range the power loss is predominantly radiation of the resonance lines and recombination on

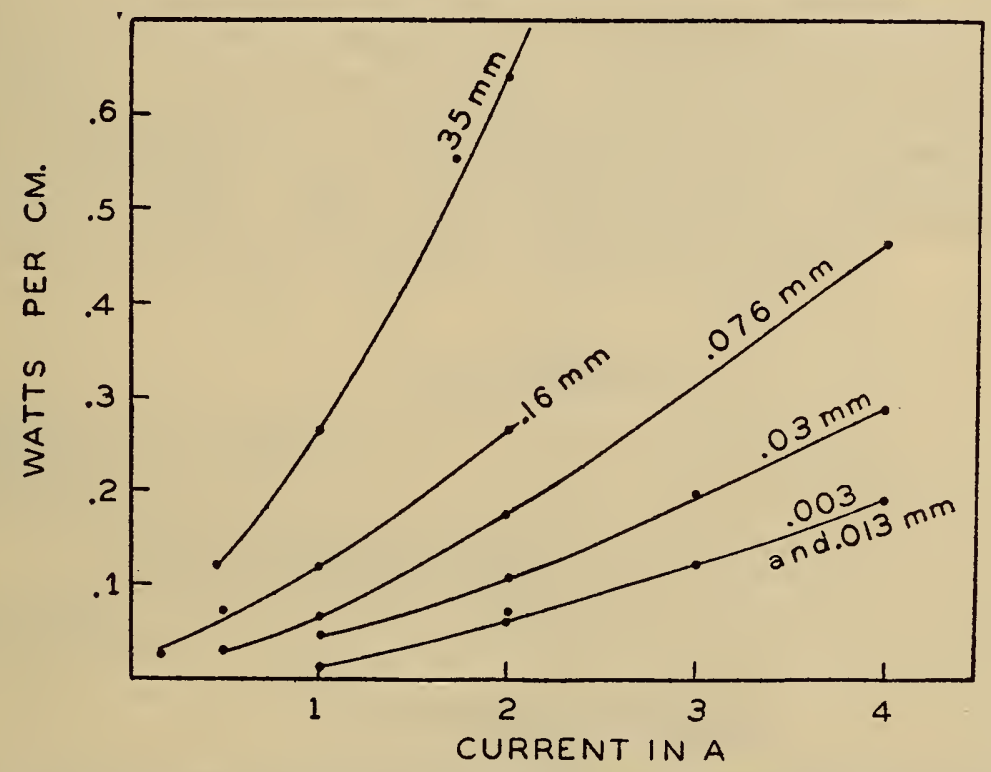

FIgURe 5.-Balance of the power loss not accounted for by recombination and radiation $(P-W-R)$ as a function of the current

the walls. It suggests that an approximate theory can be developed which considers production of ions and of the first excited state exclusively and that other phenomena can be treated independently. In this connection it is of interest to note that the visible spectrum of cæsium involving higher excited states shows large variations in intensity and intensity distribution in contrast to the very gradual change of the total radiation which is largely resonance radiation.

The published figures on the radiation efficiency of the Osram sodium lamp referred to in the introduction are consistent with our results, though the data refer to the entire discharge rather than the - positive column, so that the values are lower. The cæsium positive column, with a current density of 1 amp. per $\mathrm{cm}^{2}$ at low pressure, radiates 28 per cent of the power and at $0.1 \mathrm{amp}$. per $\mathrm{cm}^{2}$ the radiation is 96 per cent as compared with the overall efficiencies of 9 and 70 per cent for the sodium discharge. Evidently the alkali discharges are qualitatively similar in their characteristics.

Alkali vapor discharges of the type used in this work may have useful applications as sources of intense monochromatic radiation. The intensity of this radiation is insensitive to pressure and current 
variations, so that a very accurate control of intensity is possible. The fact that the efficiency of production of radiation can be made almost unity is of little practical value, since the high efficiency is attained only by keeping the power input and brightness low.

Washington, April 11, 1932. 